Volume 1

Issue 1 -- Melanoma Research

\title{
Proceedings of 2013 Aurora Scientific Day
}

Follow this and additional works at: https://aurora.org/jpcrr

Part of the Medical Education Commons

\section{Recommended Citation}

Proceedings of 2013 Aurora Scientific Day. J Patient Cent Res Rev. 2014;1:48-62. doi: 10.17294/2330-0698.1012

Journal of Patient-Centered Research and Reviews (JPCRR) is a peerreviewed scientific journal whose mission is to communicate clinical and bench research findings, with the goal of improving the quality of human health, the care of the individual patient, and the care of populations.

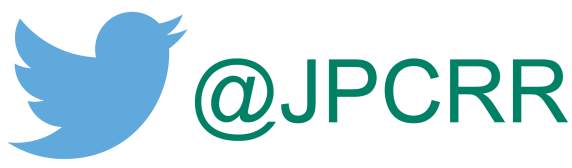


The following abstracts were presented as part of the 2013 Aurora Scientific Day program. All authors have an affiliation with Aurora Health Care, unless otherwise noted.

\section{Clinical Approach to Non-Resolving Pneumonia: A Survey of Wisconsin Primary Care Clinicians \\ Louks H, BS, UW Department of Family Medicine \\ Fixmer J, MD, Formerly Department of Family Medicine \\ Baumgardner DJ, MD, Department of Family Medicine, Aurora UW Medical Group/Center for Urban Population Health}

Background/significance: Little has been reported regarding the approach of primary care clinicians to ambulatory, non-responsive pneumonia (ANRP), variously defined as pneumonia which has not improved with 3-10 days of antibiotic therapy. Anecdotal experience suggests that changes in antibiotic prescription may occur without diagnostic testing for uncovered etiologic agents such as fungi (estimated 1-7\% of pneumonia in Wisconsin).

Purpose: To determine the stated approach to this clinical problem by primary care clinicians.

Methods: A survey containing an algorhythmic, scenario based clinical case of ANRP was sent electronically to the 103 eligible members of the Wisconsin Research and Education Network Survey Group of Wisconsin area primary care clinicians. Respondents were presented with potential diagnostic and therapeutic responses to the case scenario which was constructed from recent consensus guidelines.

Results: Surveys were returned from 53/103 members of which 44 were completed (61\% male, 30\% rural, mean 20 years practice, 13 counties). X-rays were "ordered" by $39(89 \%)$ at initial presentation, and all 39 ordered antibiotics (70\% azithromycin). After 4 days of no response, 25/39 (64\%) did additional diagnostic testing, 26/39 (67\%) changed antibiotics (5 without further testing); after 11 days of no response 3/39 (59\%) ordered CT, 16 (41\%) bronchoscopy and 28 (72\%) either CT or bronchoscopy. Five clinicians (11\%) did not order initial X-rays ( $3 / 5$ started antibiotics), nor did they order further testing after 4 days of no response. Overall, $29 / 44(66 \%)$ had done no sputum or antigen testing for bacteria by 4 days, and 24/44 (55\%) had done no specific testing for fungi by 11 days of ANRP.

Conclusion: When presented with an ANRP scenario, a majority of regional primary care clinicians both change antibiotics and do further testing after 4 days of no response, but do not test for fungi by 11 days. These findings highlight the need for practice guidelines based on research outcomes and expert experience to establish pathways for optimal treatment of ANRP. Studies are underway to determine what is done in actual practice.

Feasibility of Using the Breastfeeding Efficacy Beliefs Inventory for Improving Breastfeeding Initiation Rates in an Urban Population

Cox Bauer CM, DO, Bose D, MD, Department of Obstetrics \& Gynecology
Bernhard KA, MPH Center for Urban Population Health

Baumgardner DJ, MD, Department of Family Medicine, Aurora UW Medical Group/Center for Urban Population Health

Background/significance: Breastfeeding is the preferred method of infant nutrition. However, initiation rates of breastfeeding at Aurora Sinai are only $56 \%$ with a goal of $82 \%$ initiation per Healthy People 2020. Therefore confidence in breastfeeding in this population is important to find the barriers that must be overcome to reach this goal.

Purpose: To assess perceived breastfeeding confidence (BFC) and determine barriers in relationship to breastfeeding (BF) in an urban population.

Methods: Study Design: The Breastfeeding Personal Efficacy Beliefs Inventory (BPEBI) was used to determine perceived BFC. The survey was distributed to 272 obstetric patients during prenatal appointments. BF initiation rate at discharge was determined by medical chart review. Data Analysis: A principal component factor analysis with Varimax rotation was used to examine the structure of the BPEBI. The criterion for the determination of the number of factors was an Eigenvalue of greater than 1 and a loading factor of .30. Demographic data were summarized using descriptive statistics. Score variables were compared with the Mann-Whitney test. Univariate regression was used to determine association of predictor variables with individual inventory scores, and multivariate models were constructed with significant variables. Results: Survey response rate was $88 \%$ (240/272). The mean age was $25.3 \pm 5.7$, median age of $24 ; 64 \%$ of individuals had a high school diploma/GED. The overall BFC was $74 \%$. In multivariate models, BFC increased with prior exposure to $\mathrm{BF}$ $(p=0.03)$, exclusively BF ( $p=0.03)$, and length of $B F(p=0.02)$. Factor analysis identified two constructs: Confidence to Manage Motivation and Confidence to Manage Technique/Environment. Within the motivation construct, BFC increased with prior exposure to BF ( $p=0.006)$ and exclusively $B F(p=0.001)$, and within the technique/environment construct, BFC increased with exclusively $\mathrm{BF}(\mathrm{p}=0.000)$. BF initiation rate at discharge was $56 \%$. Conclusion: These women rated themselves as confident with breastfeeding, in contrast to the $\mathrm{BF}$ initiation rate at discharge. The two main barriers to increased BFC were not having prior exposure to BF or not exclusively breastfeeding.

\section{My Chrysosporium is a Scedosporium}

Baumgardner DJ, MD, Department of Family Medicine, Aurora UW Medical Group/Center for Urban Population Health

Chaturvedi S, PhD, Wadsworth Laboratory, New York State Department of Health

Li X, MD, Wadsworth Laboratory, New York State Department of Health

Background/significance: A mold which phenotypically resembles and has the same unusual ammonia tolerance as Blastomyces dermatitidis was previously isolated in-vitro from soil. It was tentatively identified as Chrysosporium zonatum. 
Purpose: To positively identify and determine the ubiquity of this organism which may be pathogenic and share a microenvironment with B dermatitidis.

Methods: The original isolate was identified by expert phenotypic analysis and internal transcribed spacer region rDNA (ITS) sequencing and BLAST search. 10 soil samples were obtained from a yard, park and riverbank in suburban Milwaukee County, November 5, $2012\left(5.6^{\circ}\right)$, diluted two-fold in aqueous 0.5\% allantoin, Tween-80 (20 ml/l) and penicillin/streptomycin and incubated at $37^{\circ}$. Three weeks later, samples were spread on low glucose, high (13.2 mM) ammonia basic salts agar plates at $\mathrm{pH}=7.5$ at $37^{\circ}$ in gas impermeable bags. New isolates were examined, and compared to the original isolate, on Sabouraud dextrose agar (SDA) at $20^{\circ}$ and $37^{\circ}$ for colony size, morphology and microscopic appearance.

Results: The original isolate was identified as Scedosporium apiospermum, a fungus associated with opportunistic, traumarelated and other human infections which has been isolated from feces, sewage, soil and other materials. A phenotypically similar strain was isolated from 3/4 samples from the yard (beneath the dryer vent that yielded the original isolate, under a woodpile and next to a sandbox). No colonies formed on plates from the other 7 samples.

Conclusion: Scedosporium apiospermum may compete with Blastomyces in high-ammonia environments, and may be specifically enriched by this 2-step soil isolation technique, suggesting the need for test modification to favor Blastomyces isolation. Ammonia tolerance may explain the finding of Scedosporium in feces and sewage.

\section{Azathioprine hypersensitivity mimicking sepsis in a patient with Crohn's disease}

Haflund O, MD and Amin P, MD, Department of Internal Medicine Iqbal A, MD, Department of Infectious Disease

Background/Significance: CASE: 68-year-old man with $\mathrm{PMH}$ of atrial fibrillation and Crohn's disease presented with two days history of fevers 102.8F, chills, nasal congestion and cough with small amount of whitish sputum. He denied abdominal pain, diarrhea, chest pant, joint pain, rash or urticaria. Recently diagnosed with Crohn's disease confirmed by colonic biopsy. Treatment was started with Azathioprine and Prednisone.On examination, patient looked ill and was having severe rigors and high grade fevers. Abdominal exam showed mild tenderness of the left lower quadrant on deep palpation without guarding or rebound tenderness and normal bowel sounds. Laboratory date showed normal white count and lactate level. Chest X-ray, urine analysis, and blood culture results were unremarkable. CT scan of the abdomen and pelvis with contrast showed mild intrahepatic biliary dilation with normal common biliary duct and gallbladder and diverticulosis without abscess or any other pathology. Suspecting an intra-abdominal abscess, treatment with Cefoxitin was started. Patient continued to have fevers and rigors after starting antibiotics. Azathioprine was then stopped suspecting a hypersensitivity reaction. Fever and rigors promptly disappeared after discontinuation of Azathioprine. Patient clinically improved and did not have any more fevers and rigors after Azathioprine was discontinued.
Purpose: Recognize a case of Azathioprine hypersensitivity mimicking sepsis in a patient with Crohn's disease.

Methods: Case report

Results/Discussion: Systemic hypersensitivity is a rare side effect of azathioprine. The common side effects include gastrointestinal disturbances, granulocytopenia and hepatocellular injury. The majority of reactions occur in the first four weeks of initiation of the treatment. Hypersensitivity should be suspected and included in the differential diagnosis if a patient experiences fever, malaise, hypotension and renal failure. The above mentioned symptoms could relate to a true adverse drug reaction in some versus exacerbation of underlying disease or sepsis in others.

Conclusion: We suspected hypersensitivity to azathioprine in this patient due to: timing of initiation of azathioprine, presence of above-mentioned symptoms and improvement after discontinuation of azathioprine. Rechallenge test may be used for confirmation. Optimally rechallenge should be done under careful supervision. Rechallenge was not conducted in this case, because it is dangerous and can lead to life-threatening reoccurrence of symptoms.

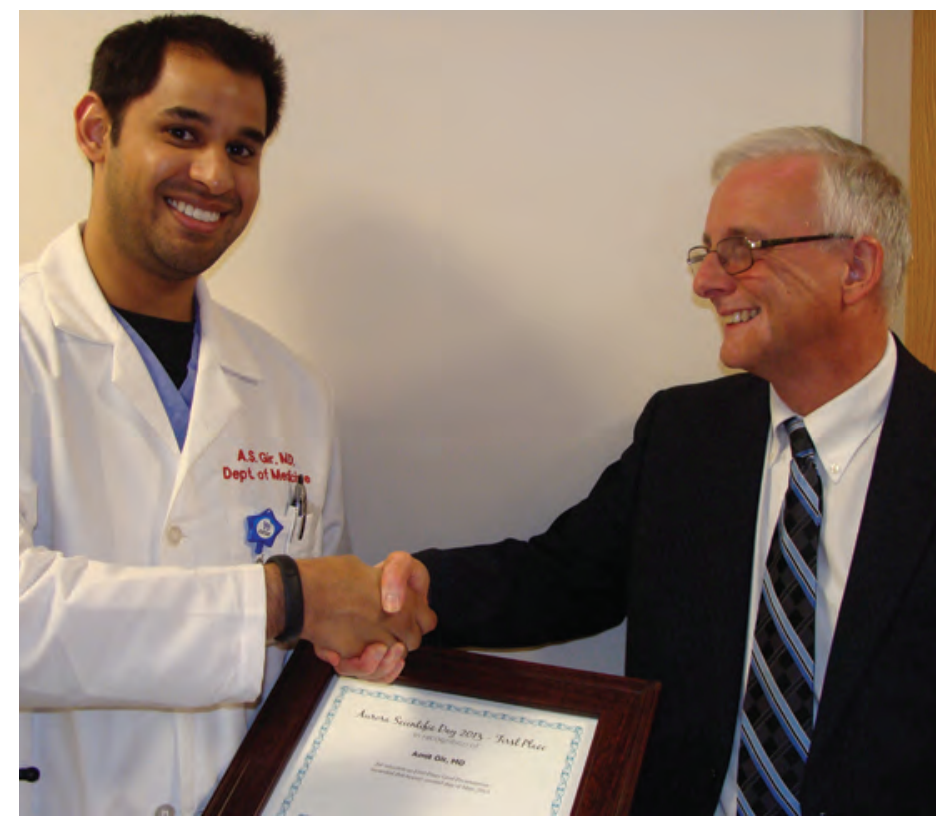

Does the Presentation of an After Visit Summary Improve Patient Satisfaction: Physician Versus Other Team Members

Bunce K, DO and Varade S, MD, Department of Family Medicine

Background/significance: Since switching over to electronic medical record system, many encounters are followed by a summarized report. These have been shown to improve patient satisfaction. Our experience at the Family Care Center is that these After Visit Summaries (AVS) often go un-reviewed by the patient. Purpose: A pilot study to investigate if the AVS is perceived by the patient to be more beneficial when presented and discussed by their physician versus being given to them by other team member (OTM).

Methods: Patients were divided alphabetically between both residents for randomization. Half were presented with the AVS by 
the physicians, half by OTM. During the next follow up visit, the patients completed a brief survey regarding their experience with their AVS at the last visit. Proportions were compared by Fisher exact test.

Results: 33 surveys were completed (19 physician-given, 14 OTM-given). Of the patients that reported a behavior change due to the AVS, $21 \%$ were presented the AVS by a physician versus $14 \%$ that were presented the AVS by an OTM $(\mathrm{p}=1.00)$. Of the patients presented with the AVS by physicians, $89 \%$ reported that they would like their AVS at future visits versus $93 \%$ of patients provided their AVS by OTM ( $\mathrm{p}=1.00)$. Due to a $47 \%$ no show rate at the clinic, lost to follow-up limited our sample size.

Conclusion: The low rate of survey completion, due in part by very high no-show rate, limited statistical comparison in this study. A 7\% improvement in effect of AVS, if substantial changes are made by patients, may be clinically significant, but would require a sample size of 1,000 to be statistically significant.

\section{Young Athlete with Bilateral Exertional Calf Pain}

Dunbar S, DO, Department of Family Medicine Englund J, MD, Orthopaedic Associates of Wisconsin

Background/significance: 15 year old male, multi-sport athlete, with one year of bilateral exertional calf pain, associated with paresthesias and no pain with rest. Neurologically intact, 2 out of 4 patellar and ankle reflexes, 5 out of 5 strength without muscle atrophy, negative slump and straight leg raise. Bounding dorsalis pedis pulse bilaterally, however with active flexion and passive dorsiflexion, pulse was not palpable.

Purpose: To evaluate the etiology and work-up of calf pain and intermittent claudication in a young athlete. In this case, the working diagnosis of popliteal artery entrapment syndrome (PAES) proved to be wrong. Therefore, clinicians should be aware of functional popliteal artery entrapment syndrome and the associated work-up and treatment.

Methods: MEDLINE search was conducted to determine the differential diagnosis of exertional calf pain in a young athlete. Additionally, specifically reviewed the presentation, diagnosis and treatment for functional popliteal artery entrapment syndrome versus popliteal artery entrapment syndrome.

Results: The differential diagnosis included exertional compartment syndrome, popliteal artery entrapment syndrome (PAES), myopathy or lumbar radiculitis. Doppler US obtained during the initial visit supported PAES given the complete cessation of flow through the popliteal arteries bilaterally with active plantar flexion. Given that MRI is the current diagnostic modality, this was obtained, however surprisingly it was negative for anatomical variants in the gastrocnemius muscles and showed no focal filing defects of the popliteal arteries bilaterally. Due to this, exertional and compartment testing was completed, which were negative for compartment syndrome. Given the results of the work-up above, the final diagnosis was functional popliteal artery entrapment syndrome.

Conclusion: There was no anatomic variation in the gastrocnemius muscles or stenosis of the popliteal arteries on MRI/MRA to support PAES. However, entrapment was demonstrated with doppler ultrasound on the initial office visit. The diagnosis of functional popliteal artery entrapment syndrome was later confirmed with
ABI testing. Going forward, is ultrasound a clinically reliable way to diagnose functional popliteal artery entrapment syndrome?

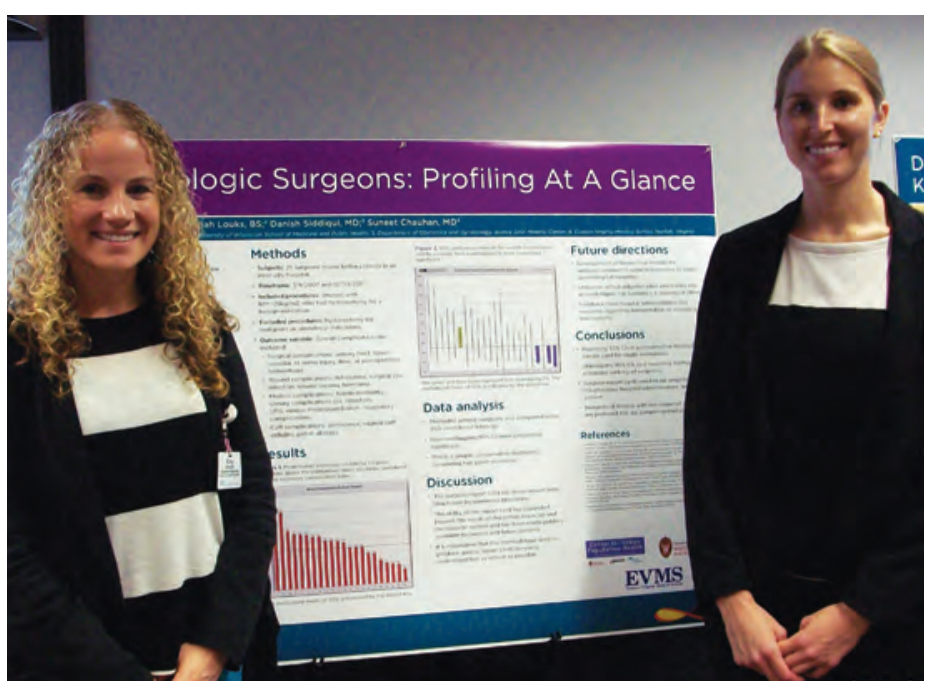

\section{Optimizing Stress Ulcer Prophylaxis in Critical Care Patients}

Katers KM, PharmD and Dalhgren MJ, PharmD, BCPS, Department of Pharmacy

Background/significance: Critical care patients are often inappropriately started on proton pump inhibitors (PPI) and histamine 2 receptor antagonists (H2RA) for stress ulcer prophylaxis (SUP), or are not re-evaluated when they are no longer indicated. This leads to overutilization in non-ICU patients and failure to discontinue prior to hospital discharge. Both agents have been associated with increased risk of nosocomial pneumonia and an association has been shown between PPIs and Clostridium difficile infection.

Purpose: Improve the appropriateness of stress ulcer prophylaxis agent use in the intensive care units.

Methods: A literature evaluation was conducted to assess qualifications for appropriate SUP. Criteria was made for appropriate SUP and approved by the hospital's Critical Care physician group. Independent indications included ventilation $>48$ hours, coagulopathy, traumatic brain or spinal cord injury, burn injury over 35\% BSA, major surgery over 4 hours, and GI bleed within the last year. Two or more of the following also indicated patients for SUP: sepsis, ICU stay $>1$ week, occult GI bleed lasting $\geq 6$ days, hepatic failure, acute kidney injury, hypotension, and steroids over hydrocortisone $250 \mathrm{mg} /$ day or equivalent. Pre-intervention data of the current pharmacological SUP use was collected in the five intensive care units (ICU) at Aurora St. Luke's Medical Center. The physician group approved a pilot where clinical pharmacists assess each patient for appropriateness of SUP and contact providers to discontinue when necessary. Pharmacists documented their interventions in a month long pilot in one of the ICU units. Following the pilot, approval was granted for pharmacists to automatically discontinue inappropriate SUP. The intervention was expanded to all of the ICU's at Aurora St. Luke's Medical Center and ultimately is to be expanded system-wide. Post-intervention was collected in April after implementation in all five hospital ICU's. 
Results: Prior to intervention, 100 ICU patients were followed and SUP use was assessed through their hospital course. Sixtythree patients were on SUP, 25 (40\%) of which were not indicated. Twenty-six (41.3\%) of the 63 patients were continued on SUP at transfer out of the ICU and $10(15.9 \%)$ were prescribed a SUP agent at discharge. In the pilot intervention, pharmacists made eleven recommendations to providers to discontinue inappropriate SUP. Ten (90.9\%) recommendations were accepted and resulted in successful discontinuation of unnecessary SUP.

Post-intervention, 91 ICU patients were followed and assessed in a similar fashion. Thirty of the 91 patients $(32.9 \%)$ were on SUP. Thirteen of these 30 patients were not indicated for the agent (43.3\%), 20 (66.6\%) continued SUP at transfer and three (10\%) were prescribed a new SUP agent at discharge. Of the patients not indicated for SUP, pharmacists automatically discontinued agents in three of the patients (23.1\%).

Conclusion: Physicians support and agree with the assessments that pharmacists make regarding the need for SUP and pharmacists are in a suitable position to assess the appropriateness of SUP and automatically discontinue the agents when they are no longer indicated. After project implementation, less SUP agents were initially prescribed by physicians in the ICUs; however, further interventions are needed to ensure that pharmacists are making proper assessments and discontinuations.

\section{The Effect of Using Guided Mental Imagery for Patients Receiving Knee or Hip Replacement}

Singh M, PhD, Patient-Centered Research

Kohler L, PT, Rehabilitation Services

Davies J, MD, Aurora Advanced Orthopaedics

Background/significance: Guided mental imagery has been described to create a sensory experience to achieve clinical goals such as to slow heart rate, stimulate immune function, reduce pain, stress, and promote physical and psychological well-being.

Purpose: The aim of this study is to assess whether the use of guided mental imagery as a therapeutic tool reduces the length of stay in inpatient care for the patients undergoing knee or hip replacement surgery.

Methods: After approval by Aurora IRB in this retrospective study, a de-identified data for all the patients who had undergone surgical procedure for knee or hip replacement performed at Aurora Sinai Medical Center from January 2009 through December 2011 were collected. Included in the study were the patients who had more than one day stay in the hospital. The study sample comprised of two groups. The guided imagery (GI) group comprised of 614 patients who used guided imagery before, during and after the surgery, in addition to the standard of care. The guided imagery intervention included the use of earphones and one of the several ways - either by CD, skylight interactive TV system, or by the download from the web site. The second group of 869 patients who received only standard of care was matched by age and gender to the GI group. Data collected included: age, length of stay, type of surgery, discharge outcome (home care, home health, skilled nursing home, and rehab facility), and the total cost for the patients.

Results: There were 924 (62.31\%) female and 559 (37.69\%) male patients who had undergone either hip $(n=559,38.37 \%)$ or knee surgery $(n=914,61.63)$. Out of the 1,483 patients, only $614(41.40 \%)$ opted for the use of the GI techniques along with standard of care. The proportion of patients opting for the use of GI was higher among females (0.44) compared to male patients (0.37, $\mathrm{p}=0.015)$. Mean number of days for the GI group were lower compared to the control group (3.21 vs. 3.36 days with $\mathrm{p}=0.047$ ). The total cost was higher $(\mathrm{p}=.0001)$ for the patients transferred to rehab facility $(\$ 22,788.95)$ compared to patients transferred either to skilled nursing facility $(\$ 17,628.73)$, home care $(\$ 16,921.93)$, or to home health facility $(\$ 16,793.86)$.

Conclusion: The study shows that the use of guided imagery as a complimentary therapeutic tool is associated with speedy recovery of patients and with the reduction of cost.

\section{Clinical Approach to Non-Responsive Pneumonia Diagnosed in the Primary Care Office: A Retrospective Feasibility Study}

Olowoyeye O, MD, Department of Family Medicine

Bernhard K, MPH, Center for Urban Population Health

Baumgardner DJ, MD, Department of Family Medicine, Aurora UW Medical Group/Center for Urban Population Health

Background/significance: Community Acquired Pneumonia (CAP) is commonly diagnosed in the primary clinical setting and often successfully treated. Treatment failures occur in $6-7 \%$ of patients treated in the ambulatory setting. However, the management of non-responsive pneumonia has been poorly studied and is not clearly understood.

Purpose: The aim of our study was to determine the feasibility of use of the Aurora data warehouse to characterize clinical features and clinician approach to NRP.

Methods: This is a retrospective study of medical records taken from the Aurora Health Care database based on ICD 9 codes for pneumonia (codes 480-486). Patient visits from 10/5/2006 to 9/30/2011 were reviewed. Our initial target sample size was 200 cases and 200 controls. We requested records seeking a clinical or radiographic diagnosis of pneumonia, which was initially made in an outpatient setting or urgent care clinic by a primary care provider. We excluded individuals who initially presented to an emergency room or specialist. Cases were those patients diagnosed with pneumonia which failed to improve within 10 days of antibiotic prescription or worsened after the fifth day following the prescription of a course of antibiotics. Controls were those with acute pneumonia, not meeting NRP definition. Variables included: race, gender, age, smoking, co-morbid conditions, duration of cough, fever, antibiotics given, change in antibiotics, and type/ duration of symptoms. Categorical data were analyzed utilizing t tests and the Mann-Whitney test; categorical variables were analyzed by chi-square or Fischer exact tests. Statistical significant was defined as $\mathrm{p}$ values less than 0.05 .

Results: Review of 339 records revealed 15 cases of NRP and 13 controls. Duplicate cases accounted for 28/339, 153 records were non-pneumonia (frequently Chlamydia [code 1 digit off]) and 12 were unknown diagnosis. Excluded pneumonia cases included 68 incomplete records and 50 specialist/ED/hospital admission. Cases were younger than controls ( 59 vs. 46 years, $p=0.02$ ). There were no other significant differences. Sample size was limiting. Conclusion: Discrepancies in diagnosis and the incomplete 
records found in the data warehouse during this time period precluded efficient obtainment of adequate sample size for this study. Use of Smart Chart for data retrieval may significantly improve efficiency. Older age is a risk for NRP in this population.

\section{An Unusual Case of Recurrent Pneumonia}

Teshome M, MD and Belete H, MD, Department of Internal Medicine

Background/significance: Bronchopulmonary sequestration (BPS) is a rare disorder of the lower respiratory tract comprising 0.15-6.4 \% of all congenital pulmonary malformations. It consists of a nonfunctioning mass of lung tissue that lacks normal communication with the tracheobronchial tree and receives its arterial blood supply from the systemic circulation. It is usually diagnosed later in childhood or adolescence after presenting with recurrent pneumonia.

Results: A 30-year-old male with $\mathrm{Hx}$ of recurrent pneumonia presented with fever, chest pain, dyspnea and non-bloody productive cough. On physical examination, he was in moderate respiratory distress. On exam, RR 24/min, PR 116/min and T $38.3^{\circ} \mathrm{C}$. Dullness on the right posterior lower lung field with inspiratory crackles was found. The rest of the examination was with unremarkable findings. Diagnostic workup: WBC 12,300 with left shift; rest of hemogram was normal. CT of chest revealed consolidation of right lower lobe with distortion of the structures and multiple air pockets. There was no extension of the tracheobronchial tree into this area. A 3-D CT reconstruction demonstrated an aberrant vessel extending from the upper abdominal aorta in to the intrapulmonary sequestration in the anterior basilar segment of the right lower lobe. There was a cystic mass with abscess cavity and thickened pleura, probably from recurrent infection. The venous drainage was in to the pulmonary vein. The patient was managed with antibiotics and open thoracotomy with right lower lobectomy and abscess drainage. Patient had smooth post-operative course and was discharged improved. No recurrence of pneumonia was reported two years after intervention.

Conclusion: This patient presented with an intrapulmonary BPS complicated by recurrent pneumonia. BPS is classified as intrapulmonary or extrapulmonary depending on the visceral pleural investment of the abnormal tissue. Communication with bronchi or lung parenchyma may be present allowing infection to occur. Resolution of infection is usually slow and incomplete due to inadequate bronchial drainage. Surgical resection is the treatment of choice for patients who present with infection or symptoms resulting from compression of normal lung tissue. Intrapulmonary lesions often require lobectomy because the margins of the sequestration may not be clearly defined. In patients who present with recurrent pneumonia at younger age, the possibility of congenital malformations like BPS should be considered and diagnostic workup pursued.

\section{Aligning Training with the 2020 Physician's Job Description}

Simpson D, PhD, Medical Education Program Director, Aurora Academic Affairs

Anderson A, MD, Sr. Vice President, Aurora Academic Affairs
Kelly C, MD, Department of Obstetrics \& Gynecology

Brill JD, MD, Department of Family Medicine

Stearns J, MD, Medical Director, Aurora Academic Affairs

Background/significance: As U.S. health care transforms, so too has the vision for medical education. Numerous publications and task force reports have called for radical reforms in medical education driven by a standardized set of core competencies identified through an analysis of physician roles and tasks. The challenge currently facing medical education is that currently the cross-cutting core competencies (e.g., performance roles and tasks) essential for our physician workforce in 2020 has not been articulated, limiting our ability to design today's training and performance-based assessments systems.

Purpose: To delineate the most likely roles/responsibilities and relative emphases for 2020 physicians to inform design, delivery and assessment of physician performance and medical education programs consistent with our future health care workforce needs.

Methods: The inter-dependency of organization, financial, and regulatory relationships guiding on today's health care transformation requires input from multiple stakeholder perspectives (e.g., health care systems leaders, medical school deans, medical education leaders) on future roles/responsibilities of physicians. A key informant methodology was selected using semi-structured protocol to obtain key stakeholder perspectives. Local and national sector informants were identified and contacted to discuss their perspectives (1-on-1 or small groups) re: "What are the most likely physician roles/responsibilities for the 2020 physician?" Consistent with constant comparative qualitative analysis methodology, the first set of 10 interviews have been completed and themes identified.

Results: Informants hold leadership positions in medical education, academic medicine and/or health care systems. The four most common role/responsibility themes emerging from analysis include the ability to: (1) work on inter-professional teams; (2) align with health system priorities (i.e., provide care in population based payment structure; (3) manage and adapt to complexity and change (including technology, continuous quality improvement); and (4) communicate well (i.e. professionalism) and provide corrective feedback to assure patient quality/safety.

Conclusion: Theme consistency across informant sectors requires significant redesign of our medical education programs to prepare the physician workforce for 2020 practice roles and responsibilities. Additional interviews will be used to affirm findings and identify specific implications for medical education redesign.

\section{Atrial Fibrillation is Associated with Increased Susceptibility of Mitochondria to Permeability Transition Pore Opening}

Emelyanova L, PhD, Ross G, PhD, Mirza M, MD, Holmuhamedov $\mathrm{E}, \mathrm{PhD}$, Jahangir A, MD, Center for Integrative Research on Cardiovascular Aging.

Background/significance: Although atrial fibrillation (AF) is known to result in progressive electrical, contractile and structural remodeling of the atria with gradual cell loss and replacement fibrosis, the molecular basis for the progressive structural alterations is not fully elucidated. Since mitochondria play an essential role 
in regulation of cell death through the opening of mitochondrial permeability transition pore (MPTP), we hypothesized that the susceptibility of mitochondria from patients with AF to PTP opening is increased contributing to the substrate that promote progression of AF to long-lasting forms.

Purpose: The aim of the study was to characterize the sensitivity of mitochondria to Ca2+-induced mPTP opening in human atria from patients with and without AF.

Methods: Freshly removed left atrial appendage tissue from patients undergoing cardiac bypass surgery with (AF) and without history of AF (nAF) was used for mitochondrial isolation. The sensitivity of mitochondria towards Ca2+-induced mPTP opening was assessed by exposing the isolated mitochondria to sequential additions of $10 \mu \mathrm{M}$ of $\mathrm{Ca} 2+$ and monitoring simultaneously abrupt mitochondrial $\mathrm{Ca} 2+$ release (Fluo-5N fluorescence), mitochondrial depolarization (Safranin O fluorescence), and swelling of mitochondrial matrix (decrease in light scattering). Differences in the expression of proteins participating in mPTP formation, including voltage-dependent anion channel (VDAC), adenine nucleotide translocase (ANT), and phosphate carrier protein (PCP) were determined by Western blotting in isolated mitochondria. The protein expression level was expressed in arbitrary units normalized to mitochondrial protein.

Results: The sensitivity of mitochondria to mPTP opening was increased in patients with AF compared to nAF ( $41 \pm 12$ vs. $66 \pm 8 \mu \mathrm{M}$ $\mathrm{Ca} 2+)$. Cyclosporine $\mathrm{A}$, an inhibitor of mPTP, increased tolerance of the mitochondria to Ca2+ loading ( $74 \pm 15$ vs. $93 \pm 9)$ and reduced mPTP opening in both group of patients. Increased sensitivity towards mPTP opening in patients with AF was associated with a 2 fold downregulation of the expression of PCP (217 \pm 66 vs. $437.6 \pm 18)$ and 2 fold increase in expression of VDAC $(428 \pm 45$ vs. 197 \pm 19 ). A trend towards increased level of expression of ANT was observed in those with AF (900 \pm 13 vs. 774 \pm 29$)$.

Conclusion: The increased sensitivity of mitochondria from AF patients to mPTP opening can be partly explained by selective downregulation of PCP protein, putative component of MPTP with upregulation of regulatory VDAC and ANT proteins.

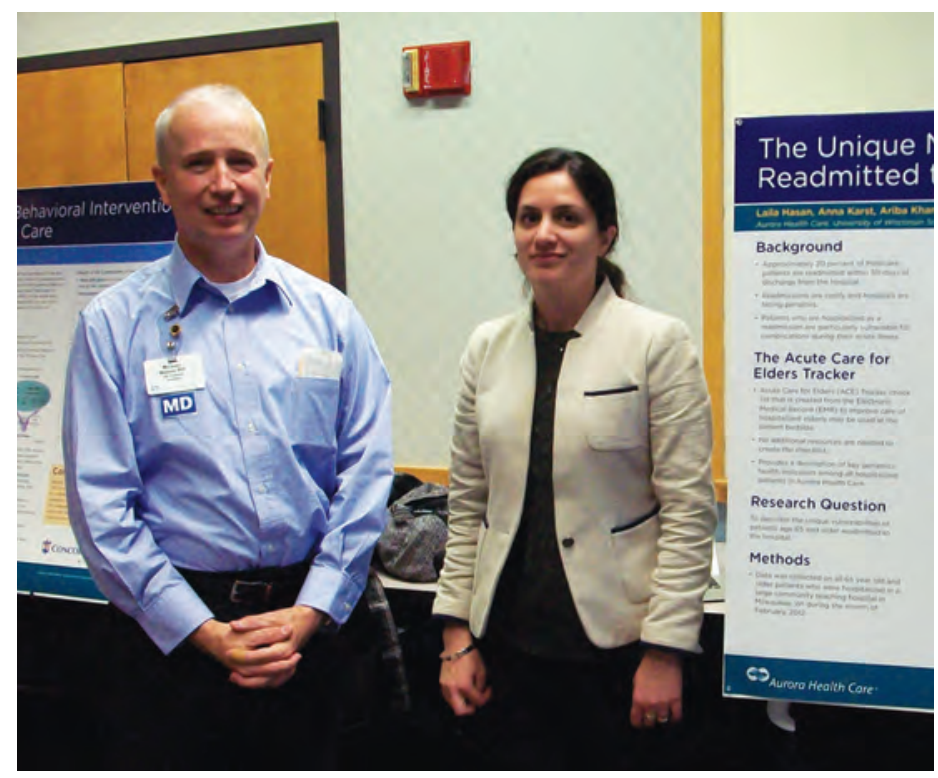

Intensive Counseling and Behavioral Interventions for Weight Loss in Primary Care

Carlton PK, DNP and Hartlaub J, DNP, Department of Family Medicine, Aurora Advanced Health Care.

Background/significance: According to the data from the National Health and Nutrition Examination Survey, 35.7\% of adults in the US are obese. In 2003, the United States Preventative Services Task Force (USPSTF) recommended clinicians screen all adults for obesity and offer intensive counseling and behavioral interventions to promote sustained weight loss. Screening and treating adults with obesity has helped attain meaningful weight loss and improve health outcomes with adequate evidence also proving better glucose tolerance and other physiological risk factors for cardiovascular disease. In 2011, Medicare approved coverage for obesity management. Medicare has approved the coverage of obesity management during primary care appointments for a year as long as a weight loss of $3.0 \mathrm{~kg}$ at the 6 month mark is achieved. Purpose: The purpose of this project was to increase the frequency of obesity diagnosis and implement intensive counseling and behavioral interventions to promote a sustained weight loss of 3.0 kg within a six month period.

Methods: This was a pilot care management quality improvement project. Five willing, older adult Medicare participants agreed to participate. Each had a BMI $\geq 30 \mathrm{~kg} / \mathrm{m} 2$. The Framework utilized was the Chronic Care Model. This guided the management of obesity. Self Determination Theory explained the motivation behind participant's healthy and effective behaviors. Helpful communication strategies included the use of 5As and motivational interviewing techniques. Participants were seen for 15 minutes each week for the first 4 weeks then every other week for 5 months. The multicomponent behavior interventional strategies included setting weight-loss goals, improving nutrition, increasing physical activity, addressing barriers to change, self-monitoring, and discussing maintenance of long term changes.

Results: Mean weight before the benefit was $187.1 \mathrm{lbs}$ or $85.1 \mathrm{~kg}$ and went down to $167.0 \mathrm{lbs}$ or $76 \mathrm{~kg}$. The mean BMI before benefit implementation was $33.03 \mathrm{~kg} / \mathrm{m} 2$ and decreased to a non-obese value of $29.68 \mathrm{~kg} / \mathrm{m} 2$.

Conclusion: High-level evidence supports offering or referring all adults with obesity for weight loss. Three kilograms of weight loss over six months can be achieved when a clinician diagnoses an adult with obesity and begins intensive counseling and behavioral interventions. This pilot project lacked diversity for age, gender, and race. Improving comfort and knowledge regarding obesity management is needed so more patients can become engaged in education for self-management.

\author{
The Unique Needs of Older Patients who are Re-Admitted \\ to the Hospital \\ Hasan M, Alverno College \\ Karst A, University of Wisconsin, Madison \\ Khan A, MD, Department of Geriatrics, Aurora UW Medical \\ Group \\ Malone M, MD, Department of Geriatrics, Aurora UW Medical \\ Group \\ Lung J, MD, Department of Geriatrics \\ Singh M, PhD, Patient-Centered Research
}


Background/significance: Approximately twenty percent of Medicare patients are readmitted within 30 days of discharge from the hospital. Patients who are hospitalized as a readmission are particularly vulnerable for complications during their acute illness. The ACE Tracker checklist is an Electronic Medical Record (EMR) report which provides a description of key geriatrics health indicators among all hospitalized patients in Aurora Health Care. Purpose: This study was designed to describe the unique vulnerabilities of patients age 65 and older who were readmitted.

Methods: Data was collected on all 65 year old and older patients who were hospitalized in a hospital in Milwaukee, WI during the month of February, 2012. To provide a convenience sample, the ACE Tracker checklist was printed every Wednesday during the one month study period. The average day of the assessment was day 7. The collected data included demographics, number of medications, functional status, and high risk medications (Beers). Readmission was noted to be present if the current hospital stay was preceded by a hospital stay in the health care system within 30 days. We compared patient characteristics between those whose current hospitalization was their second within 30 days and those whose hospitalization was not a re-hospitalization (index hospitalization).

Results: One thousand three hundred and five patients were included in the study. The average age of the patients was 77 years. For 217 patients (16.6\%) the hospitalization represented a re-hospitalization. The cohort who were receiving care during their second hospitalization were prescribed more medications when compared to those who were on their index hospital stay (13.2 vs. 11.8; $p=0.00$ ). Patients whose hospitalization represented a re-hospitalization were more likely to be on high risk medications (20\% vs. 15\%; $p=0.027$ ). Patients whose hospitalization represented a second hospitalization had a functional decline when compared to those who were on their index hospital stay $(14.7 \%$ vs. $9.7 \%$; $=0.025)$.

Conclusion: Patients whose hospitalization was a re-hospitalization were prescribed more total medications, more high risk medications, and had a decline in functional status. Further study could define if older patients who are readmitted are more vulnerable to drug drug interactions or adverse drug events.

\section{Outcome of Different Routes of Hysterectomy by Uterine Weight in Overweight and Obese Patients}

Salvo N, MD, Department of Obstetrics \& Gynecology

Siddiqui DS, MD, Department of Obstetrics \& Gynecology

Bernhard K, MPH, Center for Urban Population Health

Chauhan S, MD, Department of Obstetrics \& Gynecology at Eastern Virginia Medical School

Chen H-Y, MS, Center for Urban Population Health

Background/significance: Hysterectomy is the second most common surgical procedure done in the United States. Obesity and uterine weight contribute to length and difficulty of surgery. There is a paucity of reports on post-operative morbidity among overweight/obese women with large uteri.

Purpose: Compare perioperative outcomes among overweight $(\geq 25 \mathrm{~kg} / \mathrm{m} 2)$ and obese $(\geq 30 \mathrm{~kg} / \mathrm{m} 2)$ women with uterine weight ( $<250$ vs. $\geq 250 \mathrm{~g}$ ) undergoing different routes of hysterectomy for benign indications.

Methods: A retrospective chart review of patients (01/2005 to $12 / 2011$ ) was conducted. Inclusion criteria were $B M I \geq 25$ and known uterine weight. Exclusion criteria were hysterectomies for malignant or obstetrical indications. Variables included demographics, indications, pathology, route, operative times, co-morbidities, length of stay, primary surgeon years of practice, reoperation, follow-up, and surgical cost. Outcomes included surgical complications (urinary tract, bowel, vascular, and nerve injuries, ileus, postoperative hemorrhage), wound complications (surgical site infection, seroma, hematoma), cuff complications (dehiscence, cellulitis, pelvic abscess), and medical complications (febrile, urinary, VTE, respiratory).

Results: 1,079 hysterectomies were performed and 78\% (828) met the inclusion criteria. 29\% (245) were overweight and 71\% (585) obese. Majority were African-American (77.4\%). 52\% (427) underwent abdominal hysterectomy (AH), 27\% (221) underwent laparoscopic hysterectomy (LH), and 22\% (180) underwent vaginal hysterectomy (VH). Indications for hysterectomy included uterine fibroids $(80.6 \%)$ and menorrhagia (87.1\%). Hypertension was found in $40.2 \%$ of the patients while $21.9 \%$ were anemic prior to surgery. $25 \%$ of patients had a prior cesarean section $(23.9 \%)$ or laparotomy (24.5\%). Comparing $<250 \mathrm{~g}$ and $>250 \mathrm{~g}$ uteri, differences in wound $(\mathrm{p}=0.047)$ and cuff $(\mathrm{p}=0.027)$ complications for $\mathrm{VH}$ and for $\mathrm{AH}(\mathrm{p}=0.017)$ were statistically significant. Taking uterine weight into consideration, operating time $(\mathrm{p}<0.001)$ for both $\mathrm{AH}$ and $\mathrm{LH}$, surgical cost $(\mathrm{p}<0.001)$ for $\mathrm{LH}$, and readmission rate for $\mathrm{VH}(\mathrm{p}=0.014)$ were statistically significant.

Conclusion: Among overweight and obese women, regardless of uterine weight, there was no difference among the three routes of hysterectomies for overall complications. Regardless of uterine weight, cuff complications ( $p=0.002)$ was highest in VH (14\%). In $\geq 250$ g, cuff complications were significantly higher in $\mathrm{VH}(27 \%)$.

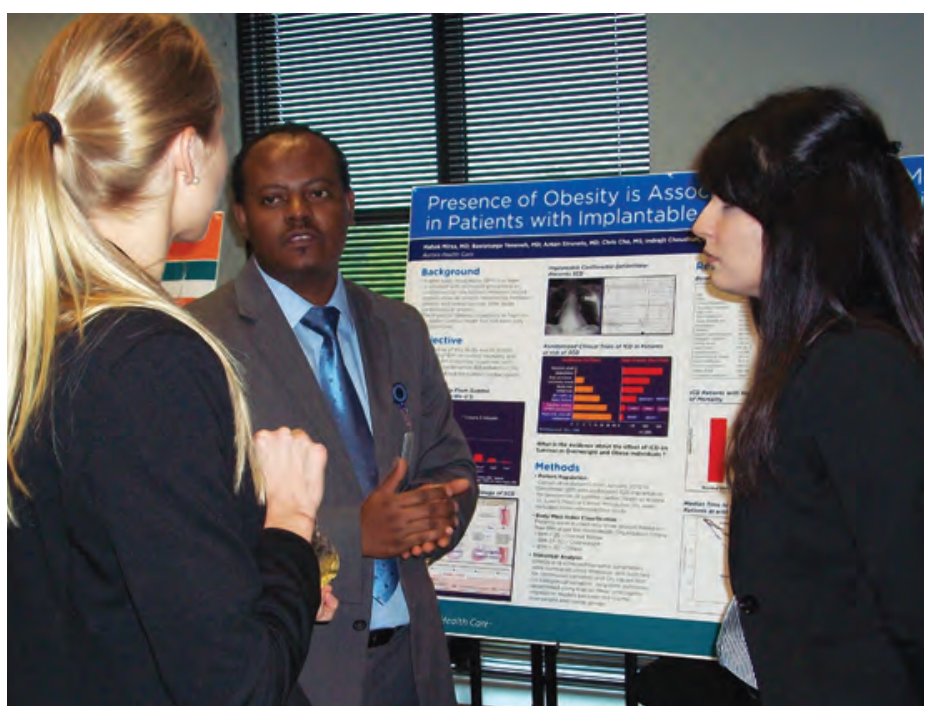

Team Competencies in Patient Care

Mehl SJ, DO, Department of Family Medicine

Anderson A, MD, Gunn L, Stearns J, MD and Simpson D, PhD, Aurora UW Medical Group.

Background/significance: Effective team functioning plays an 
integral role in patient experience and resultant scores. Several initiatives across Aurora Health Care address quality, patient satisfaction and communication but none have explicitly addressed the competencies required to be effective team members.

Purpose: Determine if implementation of TeamSTEPPS (TS), an evidence-based teamwork system, curriculum training to all caregivers will result in improved patient experience metrics and caregiver satisfaction; and if the 55-item TS assessment can be shortened without significant loss of content.

Methods: The TS team assessment, a Likert scale questionnaire, was administered to all caretakers prior to training at two Family Medicine residency ambulatory clinics. The TS curriculum was customized for initial caregiver training based on the pre-assessment results and needs of the pilot sites, and delivered following a "train the trainer" approach. Caregivers were taught core team skills and site specific skills during a 4-hour session. Item responses to the 55-item pre-questionnaire ( $\mathrm{N}=50 / 80)$ were used for cluster analysis using MINITAB software. The entire set and the large cluster 1 was subjected to principle component factor analysis, including Scree plot, with Varimax rotation. The resulting 17 questions and three retrospective questions based on "commitment to change" were administered 2 months after training ( $\mathrm{N}=55 / 80)$. CGCAPS metrics were utilized. Chi-square test with Yates correction was used for 2x2 tables of dichotomized responses.

Results: Factor analysis/Scree plot of the pre-questionnaire responses indicated 2 factors. Factor 1 (team member characteristics) explained 36\% of the variance and Factor 2 (team leader characteristics) explained $24 \%$ of the variance. When Likert scale responses were dichotomized, 2/20 questions improved after training alone $(\mathrm{p}<0.05)$. These questions included "team has adequate skills and member resources to achieve its goals” and "I make a focused effort to communicate better." Merged CGCAPS Scores in service impact, provider, provider communication and office staff all improved post vs. pre-training (11-57 percentage points).

Conclusion: Here, the 55-item questionnaire could be significantly shortened, still convey general content, and improve response rate. TS training alone did not show significant change in perceived team behaviors, yet metrics all showed improvement. Given concurrent initiatives, it cannot be concluded that this improvement is solely a result of TS training.

\section{Major Complication Rate After Mastectomy With Implant Reconstruction in a Community Hospital Setting}

Owens WL, MD, Comprehensive Breast Care Center, Aurora BayCare Medical Center

Background/significance: Mastectomy with implant reconstruction is offered to most patients with early stage breast cancer, and is sought by many desiring risk reduction mastectomy. An increased risk of major complication in mastectomy with reconstruction over mastectomy alone or lumpectomy with radiation is acknowledged, but is dependent on the breast center: technique and experience of breast and plastic surgeons, choice of implant over autologous reconstruction, and radiation oncologist opinion regarding postmastectomy radiation.
Purpose: Calculate the incidence of major complication after mastectomy with implant reconstruction at Aurora BayCare Medical Center (ABMC). Determine the rate at which postmastectomy radiation is performed at ABMC. Examine the influence of post-mastectomy radiation on the major complication rate. Compare ABMC complication rates with published results from major medical centers. Consider ways to reduce the major complication rate at ABMC.

Methods: Retrospective record review from 8/2009-1/2013 of all mastectomies with immediate implant reconstruction performed at ABMC. Major complication defined as implant loss, with or without attempts at further reconstruction.

Results: 137 implant reconstructions were performed at ABMC in 86 patients with a median follow-up of 21.3 months. Reconstruction was performed after cancer surgery in 77 cases, and after risk reduction surgery in 60 cases. Mastectomy was skin sparing in 74 cases, and nipple sparing in 63 cases. When reconstruction was done after cancer surgery, 31.2\% had radiation. After reconstruction, overall 16\% had major complication. Rate was $5 \%$ after risk reduction surgery, and $24.7 \%$ after cancer surgery. Of those cancer patients who received post-mastectomy radiation, $41.7 \%$ had major complication; of those who did not receive radiation, complication rate was 17\%. ABMC major complication rates compared favorably with those published from major centers, but post-mastectomy radiation rate was higher at ABMC.

Conclusion: Though comparable to major centers, implant loss after mastectomy with immediate reconstruction is not uncommon at $\mathrm{ABMC}$, and especially when the mastectomy is performed for cancer, and remarkably high if post-mastectomy radiation is performed. ABMC clinicians can consider methods to reduce the major complication rate while following NCCN management guidelines: improved surgical margins after mastectomy, higher threshold for recommending radiation after mastectomy, and use of autologous reconstruction after radiation.

\section{Gynecologic Surgeons: Profiling at a Glance}

Bernhard KA, MPH, Center for Urban Population Health

Siddiqui DS, MD, Department of Obstetrics \& Gynecology

Louks HA, BA, University of Wisconsin School of Medicine and Public Health

Chauhan SP, MD, Division of Fetal Maternal Medicine at Eastern Virginia Medical College

Background/significance: Surgeon report cards have become a popular approach to surgery quality improvement. Careful consideration must be taken when presenting data on surgeon performance to prevent consumers from drawing incorrect conclusions. The majority of the research on surgeon profiling has occurred in cardiac surgery. From the Cardiac surgery literature, three mechanisms of reporting surgeons; complications are readily used: means, 95\% CI, and hierarchical logistic modeling (HLM). There is paucity of studies within the specialty of gynecology.

Purpose: To evaluate the importance of utilizing 95\% confidence intervals (CI) in reporting complication rates of gynecologic surgeons.

Methods: Surgeons who performed a hysterectomy from July 2007 to December 2011 on women with BMI $\geq 25 \mathrm{~kg} / \mathrm{m} 2$. Hysterectomy 
performed for malignant and obstetrical indications were excluded. The outcome of interest was the overall complication rate which included the surgical, wound, and medical complications. Nonoverlapping $95 \%$ CIs were considered significant.

Results: During the four-year study period, 538 hysterectomies were performed by 25 surgeons. The cohort of surgeons had an institutional complication rate of $25 \%$. Comparisons of the means indicated that one surgeon (S1) was an outlier and had significantly higher overall complication rate of $60 \%$. When utilizing the 95\% CI method, one surgeon (S6) had a 95\% CI (25.1-45.4\%) that did not fall within the institutional mean (25\%) which was significantly different from the CIs of three surgeons, S19 (95\% CI: 6.6-23.2\%), S21 (95\% CI: 5.0-23.3\%), S22 (95\% CI: $1.8-23.1 \%$ ). The remaining 21 surgeons had similar complication rates and $95 \%$ CIs that fell within the institutional mean.

Conclusion: Reporting the $95 \%$ CI, as opposed to other statistical analyses, allows for greater transparency due to its simplicity and convenience. Utilizing the $95 \% \mathrm{CI}$ as a reporting method eliminates ranking of surgeons.

\section{Racial Disparities in Graft Loss Following Kidney Transplantation - A Single Center Experience}

Nilakantan V, PhD, Last B, BS, Singh M, PhD, Dalmar A, MD, Shi $\mathrm{Y}, \mathrm{PhD}$, Patient-Centered Research

Background/significance: Although national success rates in kidney transplantation are relatively high, the survival rate in African Americans (AA) drops progressively (1 year$89.9 \%$, 3 year- $72.9 \%$, 5 year-58.65\%) compared to Caucasians (1 year-92.3\%, 3 year-83.8\%, 5 year-75.5\%). Since 1998, Aurora St. Luke's Medical Center (ASLMC) has performed 317 kidney transplantations, however the survival data and variables affecting graft outcomes across different racial groups is unclear.

Purpose: The goal of this study was to compare survival rates and graft function across different racial groups following kidney transplantation at ASLMC.

Methods: This study was approved by the Aurora Institutional Review Board. A comprehensive retrospective chart review of all adult kidney transplant patients $(n=209)$ from 1999-2009 was performed. Patients that had more than one transplant were excluded from the study. Patient information included demographics, BMI, cardiovascular risk factors, time on dialysis and cold ischemia time, date of rejection, medication adherence, lab analyses and date of death. Descriptive statistics and uni-variate analysis was performed with statistical significance set at alpha $<0.05$. Time to event analyses included patients' survival as primary outcome and graft loss as secondary outcome. Significant predictors of graft loss were evaluated by logistic regression modeling.

Results: Out of 209 patients, there were 34 AA, 43 other (32 Hispanic and 12 Asian) and 132 C. While the distribution of demographic variables were similar across all racial groups, the mean time to dialysis for AA was slightly higher compared to $\mathrm{C}(\mathrm{p}=0.057)$. AA had lower 5 year survival $(85.7 \%)$ compared to $C(93.2 \%)$ but this was not statistically significant $(p=0.141$ ). At 5 years post-transplant, graft function was significantly lower $(\mathrm{p}=0.016)$ in AA compared to $\mathrm{C}(77.1 \%)$ vs. C $(92.4 \%)$.
Although there was no single independent contributor identified to graft loss, logistic regression modeling showed that AA who had low medication compliance also had the lowest graft survival $(p=0.030)$. Correspondingly, 3 year serum prograf levels were also significantly lower in AA compared to $\mathrm{C}(\mathrm{p}=0.038)$.

Conclusion: In this retrospective study, AA had higher graft loss following kidney transplantation, although the reasons are not very clear. Race and medication non-compliance are both possible factors that could affect graft survival indicating the need for larger prospective interventional studies to improve medication adherence.

\section{Adherence to Standard of Care for Patients who Underwent Endovascular AAA Repair Procedure}

Cho C, MS, Patient-Centered Research

Simmons JA, RN, BSN and Farrell J, RN, BSN, Cardiovascular Data Management.

Mewissen MS, MD, Aurora Cardiovascular Services

Background/significance: Endovascular approach to abdominal aortic aneurysm (AAA) repair has been shown to have equal efficacy with regards to long-term survival as open surgery, without the inherent risk and long recovery time associated with open surgery. However, studies have found that endovascular AAA patients are significantly more likely to require re-intervention and have known post-operative complications, namely endoleak type II. Therefore, the ACCF/AHA guidelines specify that endovascular treatment should only be used for patients who meet the anatomical criteria and those who can comply with follow-up imaging requirement. Specifically, the standard of care guidelines includes follow-up visits with CT scan at 1 month, 6 months and then annually post procedure.

Purpose: The purpose of the study is to assess whether these ACCF/AHA guidelines for patient follow-up are being followed. In addition, we will evaluate whether there are differences in the characteristics of patients who receive standard of care following endovascular AAA repair and those who do not.

Methods: This is a retrospective study of the 239 patients who underwent endovascular AAA repair at various hospitals within Aurora Health Care from April 1, 2011 to October 31, 2012. The outcome of interest is whether patients received their 1st follow-up visit with an accompanying CT scan within 6 weeks of their procedure, which is more relaxed than the 1 month requirement. Chi-square tests and hierarchical logistic regression were used for univariate and multivariate analysis, respectively.

Results: Overall, 122 out of the 239 (51\%) of the patients received a follow-up visit with CT within 6 weeks of their procedure. The results of the analysis found that physicians were a significant factor in whether patients get their required follow-up in time $(\mathrm{p}=.0039)$. In addition, patients older than 75 years of age $(p=.0024)$ were found to be significantly less likely to have received their follow-up visit within 6 weeks of procedure ( $\mathrm{p}=.0015)$.

Conclusion: Endovascular approach to AAA repair requires surveillance and timely long-term follow-up. However, this study found that less than half of the patients are receiving their follow-up in the recommended ACCF/AHA timeframe. The physicians, the age of the patients, no endoleak at completion and distance to site 
are important factors in whether patients get their follow-ups. Therefore, physicians need to be more mindful about ordering follow-up and older patients and their families will have to be better educated about the need to adhere to their follow-up schedule.

\section{Assessment of Resident Knowledge and Comfort with Menopause and Openness to Integrative Therapies: The Short Term Impact of Evidence Based Menopause Hand-Out}

Handler H, MD, Department of Family Medicine

Bernhard KA, MPH, Center for Urban Population Health

Baumgardner DJ, MD, Department of Family Medicine, Aurora

UW Medical Group/Center for Urban Population Health

Background/significance: Residents may not receive adequate training regarding menopause, may not be open to integrative therapies, or may prefer conventional therapies despite adequate evidence for the former.

Purpose: To assess resident knowledge, comfort and confidence with menopause and their openness to integrative treatments and determine the short term impact of a patient hand-out.

Methods: We developed an integrative evidence-based patient menopause hand-out, then distributed a survey to 8 OBGYN and 14 Family Medicine residents before and after respondents read it. The survey assessed factual knowledge of menopause (5 items) and asked 4 Likert scale questions regarding comfort, confidence and openness to integrative treatment and life-style modifications. Paired variables were analyzed using McNemar test (nominal data) or Wilcoxon Signed Rank Test (Likert scales). Unpaired variables were analyzed using Chi-Square or Fisher exact test (for nominal data) or Mann Whitney test (Likert scales).

Results: There were 22 respondents, 16 females and 6 males. The median pre-handout survey rating of resident comfort and training preparation regarding menopause symptoms was 2.0/5.0 and 3.0/5.0, respectively. Overall, 2/5 knowledge questions regarding integrative treatment of menopause improved after reading the hand-out $(\mathrm{p}<0.001)$, as did comfort treating menopause symptoms $(\mathrm{p}=0.001)$, and score on a question indicating willingness to use integrative modalities $(\mathrm{p}=0.014)$. The pre-handout survey indicated that OBGYN residents are significantly less willing to use integrative modalities than Family Medicine residents $(\mathrm{p}=0.001)$. There were no resident gender or other specialty differences in responses, however sample size limited analysis.

Conclusion: Residents perceive they are not receiving adequate training in integrative menopause therapies, which is a concern given that up to $75 \%$ of menopausal women have symptoms. Residents' level of confidence significantly improved after an evidence based hand-out was reviewed. OBGYN residents may be inherently less willing to use integrative modalities for menopause symptoms. Future studies could assess what is the best way to supply this knowledge in residency so that residents are not resistant to integrative therapy, and that their comfort level is enhanced.

Breast Atypia: Timing of Progression to Subsequent Clinically Significant Event

Tjoe JA, MD, Surgical Breast Oncology
Singh M, PhD, Patient-Centered Research

Last B, BS, Patient-Centered Research

Neitzl GF, MD, Department of Pathology

Marx JJ, PhD, Patient-Centered Research

Jewett C, BS, Patient-Centered Research

Background/significance: Atypical hyperplasia (AH) increases a woman's risk for breast cancer. This can cause significant anxiety, leading women to choose risk reduction options of tamoxifen and/ or prophylactic mastectomies.

Purpose: Understanding the natural history of AH and subsequent breast cancer may help patients select and time options for risk reduction.

Methods: Aurora Health Care's experience was retrospectively reviewed for women initially diagnosed with pure breast $\mathrm{AH}$, either atypical ductal hyperplasia (ADH) and/or atypical lobular hyperplasia (ALH). 31,921 patients with the ICD-9 code of 610.8/.9 (other specified or unspecified benign breast disorder) were screened between 1/1995-12/2010. After elimination of repetitive diagnoses from multiple visits, non-breast disease, non-atypical benign breast disease, and previous diagnosis of breast malignancy, the study cohort consisted of 370 breasts with a principal diagnosis of pure AH involving 360 patients. Slides were reviewed by a single pathologist, blinded to the original diagnosis. Those patients who progressed to a subsequent clinically significant event (SCSE), i.e., another diagnosis of $\mathrm{AH}$ (increasing the calculated Gail Model 5-year and lifetime risk of breast cancer) or malignancy were analyzed for race, age, SCSE pathology, laterality, medical/surgical risk intervention, and time to progression.

Results: 31(8.61\%) patients developed a SCSE. Mean age at initial diagnosis of AH was 57.9 years (SD 10.6, range 29-84). Racial makeup was similar to controls: 27 (87.1\%) Whites, 3 (9.6\%) Blacks. 18 (58.1\%) developed the SCSE within the ipsilateral breast, and $13(41.9 \%)$ within the contralateral breast $(\mathrm{p}=0.369)$ whether the initial diagnosis was ADH, ALH, or mixed ( $\mathrm{p}=0.405$ ). Regarding histology of the 2nd event, 14 (45.2\%) were diagnosed again with AH, 5 (16.1\%) progressed to carcinoma in situ (CIS), and $12(38.7 \%)$ progressed to invasive carcinoma (IC) $(\mathrm{p}=0.115)$. Resection of the AH did not reduce the chance of SCSE within the ipsilateral breast $(p=0.924)$. Of those 31 who developed a SCSE, $3(9.7 \%)$ had taken tamoxifen, which did not influence subsequent histology. Time to development of recurrent AH, CIS, and IC was $2.87,2.64$, and 5.12 years, respectively $(\mathrm{p}=0.038)$, uninfluenced by type of atypia diagnosed $(\mathrm{p}=0.595)$.

Conclusion: Time to progression from AH to IC, in our series, is approximately 5 years. However, we cannot identify which patients with AH will develop malignancy, highlighting the need for prognostic biomarkers for more effective risk stratification.

\section{Evaluation of Sleep Inducing Medications as a Contributor to Adverse Events in the Elderly}

Kaiser ML, PharmD and Halsey J, PharmD, CGP, BCPS, Department of Pharmacy

Background/significance: Benzodiazepines, first generation antihistamines, sedative hypnotics, and tricyclic antidepressants 
are often used for inducing sleep. Patients 65 years and older are at an increased risk of falls, delirium, cognitive decline, and functional decline, and in some instances, mortality when receiving these medications. The FDA recommends reducing zolpidem doses as blood levels may be high enough the morning after use to impair activities requiring alertness. Alternative therapies may be used as substitutes for medications in promoting sleep in the elderly.

Purpose: The study's purpose was to develop a behavioral intervention protocol that reduced the use of sleep medications in the elderly, thereby increasing patient safety and decreasing related adverse events.

Methods: Retrospective fall and medication administration data was examined for inpatients from August 2011 through December 2012. The incidence of delirium in hospitalized elderly patients was also examined during this timeframe. An alternative sleep protocol utilizing nonpharmacologic sleep alternatives was created and implemented on the ACE floor with the help of a multidisciplinary team.

Results: Thirty patients were eligible for study inclusion. Seven patients (23.33\%) used a target medication before admission and 27 patients $(90 \%)$ were prescribed a target medication during hospitalization. Twenty-six patients $(86.67 \%)$ were placed on the alternative sleep protocol and two patients successfully used these alternative methods. Four patients (13.33\%) received at least one dose of zolpidem after placement on the alternative sleep protocol. An average of 30.67 doses of zolpidem were prescribed and administered to patients 65 years and older monthly at baseline compared to 8.67 doses during the study. The average monthly falls in patients 65 years and older receiving a target medication at baseline was 0.35 and during the study was 0.67. At baseline, the monthly average delirium incidence in patients 65 years and older was $15.06 \%$ and the monthly average incidence of functional decline in these patients was $15.18 \%$. Study period delirium and functional decline data continue to be collected.

Conclusion: While current data does not support the use of nonpharmacologic sleep alternatives to reduce falls, delirium, or functional decline, the study may have been underpowered to detect a difference. Prescribing and administration of sleep induction agents declined significantly compared to baseline.

\section{TGF-beta1 Induced microRNA-21 (miR-21) Up-Regulation in Human Cardiac Fibroblast Precedes Their Transformation to Myofibroblast}

Rizvi F, PhD, Ross G, PhD, Holmuhamedov E, PhD, Mirza M, MD, Jahangir A, MD, Center for Integrative Research on Cardiovascular Aging

Background/significance: The prevalence of atrial fibrillation (AF) increases with aging and aging-associated diseases. Although a common denominator underlying the substrate for aging-related $\mathrm{AF}$ is excessive extracellular matrix deposition, the molecular mechanisms regulating human atrial fibrosis are not fully understood. MicroRNAs (miRNA) are small noncoding regulatory RNAs that control gene expression by translational suppression and disruption of target mRNAs. Increased expression level of atrial microRNA-21 (miR-21) has been demonstrated in animal models and patients with AF with positive correlation with atrial collagen content. However, the time course of miR-21 elevation and fibroblast activation that results in collagen deposition in patients at risk for AF has not been characterized.

Purpose: The aim of this study was to delineate the temporal association of changes in the cytokine-induced expression of miR-21 to activation of fibroblast isolated from patients with no history or risk factors for AF, with risk factors, but no AF, and those with AF

Methods: Passage 3 cultured human cardiac fibroblasts isolated from patients with no history of or risk factors (hypertension, heart failure, valve disease or age $>45$ years) for AF (Group 1 ), with risk factors but no history of AF (Group 2) and those with AF (Group $3)$ were used for this study. The effect of TGF-beta1 $(5 \mathrm{ng} / \mathrm{ml})$, a central pathological mediator of fibrosis, on temporal expression of miR-21 and transformation of fibroblast to myofibroblast was determined using Real-time PCR with miR-21 primers and immunohistochemistry with antibodies against Vimentin as a marker for fibroblast and alpha-smooth muscle actin (alpha-SMA) for myofibroblasts. MiR-21 expression and number of alphaSMA+ cells was compared between TGF-beta1 untreated and treated fibroblasts.

Results: TGF-beta1 exposure increased expression of miR-21 and the number of alpha-SMA+ cells in all groups. In fibroblasts from patient with no history of or risk factors for AF the level of expression of miR-21 reached a peak (350\% of baseline) within 24 hours of TGF-beta1 treatment, followed by a time-dependent increase in the mRNA expression of alpha-SMA that reached a peak (400\% of baseline) in 48 hours. The baseline protein expression level of alpha-SMA in unstimulated fibroblast isolated from patients with persistent AF was 1.5 fold higher than those with no history of AF and 3.0 fold higher than those with no risk factor or history of AF with relatively smaller increase in alphaSMA expression following TGF-beta1, suggestive of endogenous activation of fibroblasts compared to those without AF.

Conclusion: The temporal relationship of miR-21expression following TGF-beta1 stimulation before fibroblast transformation to myofibroblast is suggestive of a regulatory role of miR-21 in fibroblast activation rather than a secondary effect of myofibroblast on miR-21 expression. Patients with persistent AF appear to have endogenously active fibroblast compared to those without risk factors or history of AF.

\section{Ciprofloxacin Enhances Matrix Metalloproteinases Activity in Human Aortic Smooth Muscle Cells}

Wang D, MD, Department of Internal Medicine, Aurora Health Care

Jahangir A, MD, Center for Integrative Research on Cardiovascular Aging

Tajik J, MD, Aurora Cardiovascular Services

Background/significance: The fluoroquinolone antibiotics have been associated with disruption of collagen tissue structures (tendon, corneal, and retina) through promotion of the expression of matrix metalloproteinases (MMPs), which promote degradation of collagen fibril. It is possible that fluoroquinolones may increase MMP activity and tissue destruction in other organs including aorta. Increased production and activity of MMPs and subsequent breaking down of collagen/elastin fibers in aortic wall plays an important role in abdominal aortic aneurysm (AAA) formation. 
However, no study has been done to evaluate the effect of fluoroquinolone on MMP activity in aortic tissue.

Purpose: We hypothesize that fluoroquinolone may induce the formation and deterioration of aortic aneurysm through promoting MMP activity and collagen degradation. Since aortic smooth muscle cells are the major source of MMPs in aortic wall, we assessed the effect of fluoroquinolone on MMP activities in human aortic smooth muscle cells.

Methods: Cultured passage 3 human aortic smooth muscle cells were subjected to total $48 \mathrm{hrs}$ treatment of Ciprofloxacin $(5 \mathrm{ng} / \mathrm{ml}$, $50 \mathrm{ng} / \mathrm{ml}$ ) or control culture media. After the treatment, culture media was collected and total MMP activities were assessed by Zymograph (10\% Gelatin gel).

Results: In cultured human aortic smooth muscle cells, Ciprofloxacin $5 \mathrm{ng} / \mathrm{ml}$ significantly increased total MMP activity by 2.2 folds $(\mathrm{n}=4, \mathrm{p}<0.05)$, and Ciprofloxacin 50ng/ml significantly increased total MMP activity by 1.9 folds $(n=4, p<0.05)$.

Conclusion: Ciprofloxacin (5ng/ml and 50ng/ml) significantly increased total MMP activity in cultured human aortic smooth muscle. $5 \mathrm{ng} / \mathrm{ml}$ is also the therapeutic concentration of Ciprofloxacin in human tissue. Our result indicated that use of fluoroquinolone antibiotics may induce proteolytic degradation of collagen structure through promoting MMP activity in aortic wall. Given the extremely broad use of fluoroquinolone and devastating outcome of AAA, it is important to assess the possible risk of aneurysm promoting effect of these antibiotics to guide antibiotic treatments.

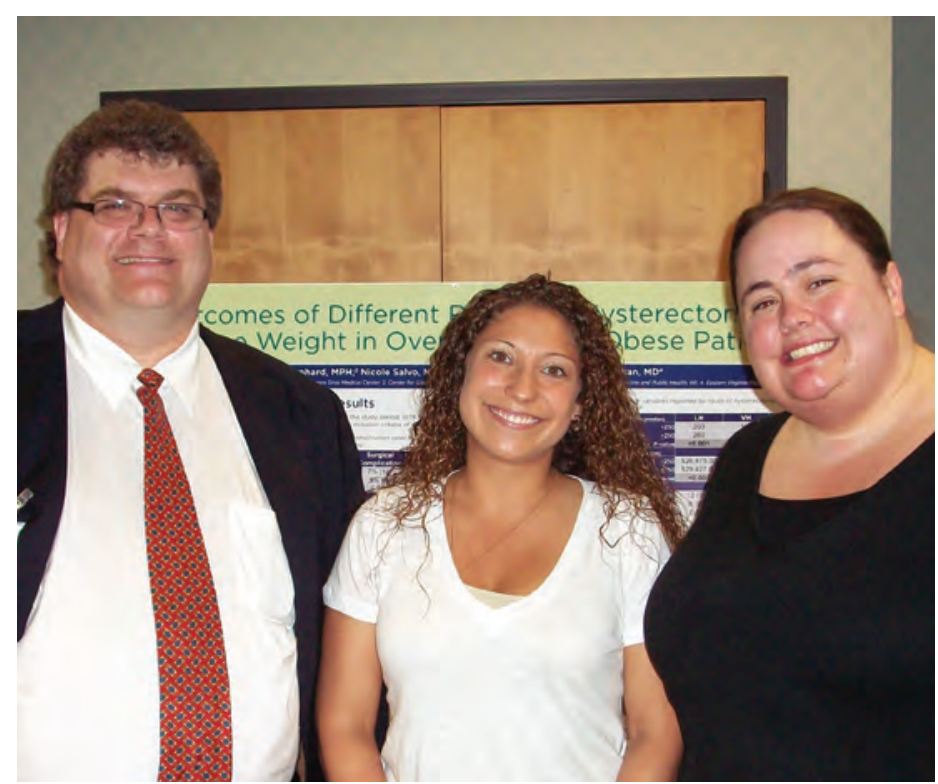

Maternal Group B Streptococcus Colonization and Neonatal Morbidity in Premature Preterm Rupture of Membranes

Hirsch DM, MD, Department of Obstetrics \& Gynecology

Bose D, MD, Department of Obstetrics \& Gynecology

Baumgardner DJ, MD, Department of Family Medicine, Aurora

UW Medical Group/Center for Urban Population Health

Garland JS, MD, Department of Pediatrics
Background/significance: Group B Streptococcus (GBS) colonization is a major cause of infectious morbidity in newborns. Purpose: Our goal was to determine if markers for neonatal morbidity are affected by perinatal maternal colonization with GBS in women whose pregnancies are complicated by preterm premature rupture of membranes (PPROM) between 24 and 34 weeks gestation.

Methods: We reviewed records of patients admitted to two urban hospitals who underwent PPROM between 24 and 34 weeks gestation between 2000 and 2011 and had a known GBS status. Associated neonatal records were then reviewed for evidence of morbidity. The primary outcome measure was Score for Neonatal Acute Physiology (SNAP). Secondary outcome measures included Apgar score $<7$ at 5 minutes, umbilical artery $\mathrm{pH}<7.1$, length of latency, and presence of sepsis, bronchopulmonary dysplasia, sepsis or neonatal death. Neonatal outcomes were compared between mothers who were GBS positive versus GBS negative. Multiple gestations and patients with significant obstetrical complications or fetal abnormalities were excluded. Categorical variables were analyzed with Chi-square or Fisher exact tests, ordinal or continuous variables by Mann-Whitney or 2-sample T-tests. Binary logistic regression was used for multivariate modeling.

Results: Of 462 patients meeting inclusion and exclusion criteria, 133 (29\%) were GBS positive and 329 GBS negative. Demographic characteristics including maternal age, race, gestational age at delivery, gravity/parity, birthweight, and route of delivery were similar by group. For the primary outcome, neonates born to GBS positive mothers trended toward higher SNAP scores than did those of GBS negative mothers (36.1\% vs. 26.1\%, $\mathrm{p}=0.07$ ). On analysis of secondary outcomes, bronchopulmonary dysplasia was present in $20 \%$ of neonates born to GBS positive and $12 \%$ of GBS negative mothers $(p=0.046)$, and this difference persisted after multivariate analysis, where gestational age was also predictive. Umbilical cord $\mathrm{pH}<7.1$ was present in $2.5 \%$ of neonates born to GBS positive and $8.4 \%$ of GBS negative mothers $(\mathrm{p}=0.049)$. There were no significant differences in Apgar score at 5 minutes $<7$, latency, presence of sepsis, or neonatal death, although Apgar at 5 minutes $<7$ approached significance $(22.6 \%$ vs. $14.9 \%, p=0.056)$.

Conclusion: Neonatal morbidity as measured by the SNAP score does not differ by maternal GBS status. However, significant differences in low umbilical artery $\mathrm{pH}$ and particularly bronchopulmonary dysplasia provoke further analysis and study.

\section{An Electronic Medical Record Marker for Delirium in Hospitalized Elderly}

Lung J, MD, Khan A, MD, Singh K, MD, Malone M, MD, Department of Geriatrics

Singh M, PhD, Patient-Centered Research

Background/Significance: Delirium is an acute confusional state that is common among hospitalized elderly and is associated with poor outcomes. This syndrome requires prompt recognition and evaluation as the underlying medical condition may be life threatening. The diagnosis of delirium is made clinically and is often under-recognized.

“Acute Care for the Elders (ACE) Tracker” is an innovative, 
automated checklist that is generated from the electronic medical record (EMR). The diagnosis of delirium is noted on ACE Tracker as being present or absent. This diagnosis is obtained from data already entered in the EMR by nurses and may be used at the patient's bedside to improve care of hospitalized elderly.

Purpose: Can the EMR checklist provide a marker for delirium?

Methods: Data was collected on all 65 year old and older patients who were hospitalized in a large, community teaching hospital in Milwaukee, WI during the month of February, 2012. To provide a convenience sample, the ACE Tracker checklist was printed every Wednesday during the one month study period. The average day of the assessment was day 7. The collected data included demographics, number of medications, functional status, and high risk medications (Beers). Delirium marker was defined as being present if there was: documentation of delirium by nurses, or administration of haloperidol within the prior 48 hours, or administration of physical restraint.

Results: One thousand three hundred and six patients were included in the study. The average age was 77 years. Two hundred and sixty patients had a marker of delirium detected on ACE Tracker (20\%). Use of Beers medications in patients with delirium marker was fifty three percent $(53 \%)$ vs. thirty three percent $(33 \%)$ in patients without delirium marker; $\mathrm{p}=0.001$. The rate of functional decline among those with delirium was $17.7 \%$ (46/260) compare to 8.7\% (91/1046) among those without delirium; $p=0.001$.

Conclusion: The EMR delirium marker may be useful in the diagnosis of delirium among hospitalized elderly patients. This may help the health care team in early recognition and treatment of delirium.

\section{TGF-beta1 Activates Mitochondrial Biogenesis and Energetic Remodeling of NIH/3T3 Fibroblasts}

Holmuhamedov E, PhD, Negmadjanov U, Rizvi F, PhD, Ross G, $\mathrm{PhD}$, Center for Integrative Research on Cardiovascular Aging

Richards J, PhD, Immunotherapy Research

Background/significance: Differentiation of fibroblasts (FB) into myofibroblasts (myoFB) is the key event in the wound healing process of damaged tissue. Following tissue injury and initial migration and intensive proliferation, FB differentiate into myoFB, cells managing remodeling of extracellular matrix and depositing collagen. Phenotypic switch from proto-myoFB into myoFB is accompanied by remodeling cell metabolism and conversion of non-excitable precursors into the contracting cells capable of synthesis and deposition of structural proteins which are orchestrated by metalloproteinase(s) into extracellular matrix. In addition to remodeling of extracellular matrix myoFB also became excitable cells, capable of mechanical contraction.

Purpose: We hypothesized that TGF-beta1 mediated differentiation of naive NIH/3T3 FB into myoFB will be associated with energetic remodeling and increased presence of mitochondria as more efficient source of intracellular ATP.

Methods: NIH/3T3 cell line obtained from ATCC. Current study used confocal microscopy, Seahorse Extracellular Flux Respirometry, multiwell plate reader and standard biochemical and biophysical protocols. Vimentin, alpha-SMA and mitochondrial proteins were determined using Western blotting;
mt-DNA was quantified with Mitochondrial DNA kit; matrix metalloproteinase(s) were determined using Zymogel.

Results: TGF-beta1 exposure induced differentiation of naïve NIH 3 T3 cells into differentiated myofibroblasts as verified by increased expression of characteristic alpha-SMA from $0.87 \pm 0.2$ to 1.44 \pm 0.27 RU (normalized to housekeeping GAPDH). At the same time, TGF-beta1 exposure increased the number of mitochondria per cell, doubled baseline respiration of differentiated cells, increased average mitochondrial DNA/cell as well as expression of mitochondria specific proteins such as ANT. TGF-beta1 exposure increased baseline respiration of NIH/3T3 cells from $1.13 \pm 0.1 \mathrm{nmol} \mathrm{O} 2 / \mathrm{min} / 106$ cells in naïve culture to $2.25 \pm 0.03$ nmol O2/min/106 cells in TGF-beta1 treated cells. Mitochondrial DNA more than doubled from $307 \pm 9 \mu \mathrm{g}$ DNA/106 cells in control to $530 \pm 12 \mu \mathrm{g}$ DNA/106 cells in TGF-betal exposed cells and was paralleled by increased expression of mitochondrial specific proteins: VDAC $58 \%$ and ANT by $57 \%$.

Conclusion: TGF-beta1 dependent differentiation of NIH/3T3 fibroblasts results in energetic remodeling and activation of mitochondrial biogenesis due to increased energy demand, which is compensated and fulfilled by increased mitochondrial biogenesis as the source of more efficient source of ATP.

\section{Resident Physician Recognition of Obesity and Patient Education in a Family Medicine Residency Clinic within an Underserved Community}

Siegel R, DO, Department of Family Medicine

Bernhard K, MPH, Center for Urban Population Health

Baumgardner DJ, MD, Department of Family Medicine, UW Medical Group/Center for Urban Population Health

Background/significance: Obesity is a major problem in Wisconsin. Primary Care Resident Physicians may not be taking the opportunity to address this medical condition and/or offering interventions.

Purpose: To assess Resident Physician recognition of obesity and acknowledgment as a medical problem and to further assess the action taken with regard to diet and lifestyle intervention.

Methods: A convenience sample of residents was selected and a brief survey was distributed to those selected at two Family Medicine residency clinics to increase awareness of patient obesity. For 8 weeks, participating residents completed the survey by writing the perceived weight, BMI, and weight category. They would add patient age, gender and resident gender prior to reviewing the patient's vitals. After reviewing the vitals, the resident would then write the actual weight and BMI. A random chart review of 100 charts was completed pre- and post-survey for the participating and non-participating (control) groups to assess obesity recognition and intervention. Pre- and post-survey comparisons utilized chi-square test or Fisher exact for categorical variables and t-test or Mann Whitney test for continuous variables. Binary logistic regression was used for multivariate comparisons. Results: There was no difference in estimated vs. actual BMI regarding patient or resident gender. As expected, BMI was a predictor of whether obesity was addressed. In the participating group there was an increased recognition of obesity among female patients $(\mathrm{p}=<0.05)$ and by female residents $(\mathrm{p}=<0.01)$. Although 
there was an increase in recognition in obesity in the post-survey participating group, there was no increase in intervention. In the control group, there was no significant change in recognition of obesity, but there was a decrease in intervention of diet, lifestyle modification and nutritional education.

Conclusion: Although there was an increase in acknowledging obesity as a medical problem following an obesity-recognition exercise, there was no increase in intervention. This increase could be secondary to physicians outside of the study having encounters with patients and adding obesity as a problem or medical history. Quality improvement measures will need to be enforced in order to better patient health and outcomes. Specific educational materials targeted toward obesity, diet and lifestyle modifications will need to be created.

\section{Weight Loss After Laparoscopic Adjustable Gastric Banding: The Role of Race, BMI and Age \\ Dalmar A, MD, Patient-Centered Research \\ Chua TY, MD, Bariatric Surgery Center \\ Singh M, PhD, Patient-Centered Research}

Background/significance: Laparoscopic adjustable Gastric Banding (LAGB) has become one of the most common bariatric surgery procedures performed in the United States since its approval in 2001. According to the latest bariatric surgery studies, there is no disparity in the use of bariatric surgery by race. However, recently, it has been hypothesized that race, BMI and age may affect the outcome after bariatric surgery. Aurora Sinai Bariatric Surgery Center of excellence, with its single surgeon, has gathered a data of 852 patients who underwent LAGB from 2001 to 2010.

Purpose: To determine the difference in mean percentage of excess weight loss (\% EWL) for 5 years after LAGB among African Americans (AA) and Caucasians.

Methods: IRB approval was obtained to conduct a retrospective review of prospectively collected data for 852 patients who underwent a LAGB from February 2001 to February 2010. Included in the analysis were only 721 patients with pars flaccida technique. The initial technique, Perigastric, was abandoned after first 90 cases because of high frequency of complications. Demographics, preoperative comorbidities, operative time (OR), length of stay (LOS) and mean \% EWL at 1, 2, 3, 4, 5 years, were compared. T-test was used to determine the mean \% EWL difference between AA and Caucasians. Univariate analysis was done to compare mean \% EWL across race (AA and Caucasians), age $(<50, \geq 50$ years) and $\mathrm{BMI}(<50, \geq 50 \mathrm{~kg} / \mathrm{m} 2)$.

Results: Data from 721 (82\% females, 78\% Caucasians) patients were analyzed. Overall AA were younger with higher BMI. Median operating room time was 59 mins for AA and 62 mins for Caucasians. Ninety-nine percent of AA and 73\% of Caucasians had 1 or more comorbidities.

The mean \% EWL was significantly higher for Caucasians compared to AA for 1 st and 2nd year intervals $(27.7 \pm 14.9$ vs. $21.5 \pm 12.2$ for first year and $38.7 \pm 19.1$ vs. $32.7 \pm 20.0$ for second year). No significant difference in mean \% EWL at 3, 4 and 5 years follow up. The only significant interaction was between age and $\mathrm{BMI}$ indicating that the mean \% EWL in the first year was higher for younger and lighter patients. There is also a trend in interaction between race and $\mathrm{BMI}$ indicating that Caucasians lost more weight than AA only if they had lower BMI.

Conclusion: Although race is a factor in determining mean \% EWL in the first and second years after surgery, the combination of BMI and age groups also may play an important role in weight loss after LAGB.

Triglycerides and Risk Factors for Heart Disease: Their Relationship with Coronary Artery Saphenous Vein Bypass Graft Occlusion

Shuaib S, MD, Murthy S, MD, Hashim H, MD, Department of Cardiology

Allaqaband S, MD, Aurora Cardiovascular Services

Cho C, MS, Center for Urban Population Health

Background/significance: Coronary artery disease prevention is achieved by controlling risk factors for atherosclerosis.

Purpose: Our objective was to test the impact of these risk factors on saphenous vein graft (SVG) occlusion in patients post coronary artery bypass graft (CABG) surgery.

Methods: We retrospectively studied 1,000 consecutive patients (mean age 66 years, 66\% men) who underwent CABG surgery from January 2000 to August 2004 for the presence of SVG occlusion on coronary angiogram. Risk factors including smoking history, dyslipidemia, obesity, hypertension and diabetes mellitus were recorded. Follow-up data were available through October 2011. Wilcoxon rank-sum and chi-square tests were used to analyze data. Results: Univariate analysis showed statistical difference in SVG occlusion for triglycerides $>150 \mathrm{mg} / \mathrm{dL}$, total cholesterol $>200 \mathrm{mg} /$ $\mathrm{dL}$, and high-density lipoprotein (HDL) $<40 \mathrm{mg} / \mathrm{dL}$ (men) or $<50$ $\mathrm{mg} / \mathrm{dL}$ (women). Logistic regression used for multivariate analysis showed SVG occlusion was strongly associated with triglycerides (odds ratio [OR] 2.2, 95\% confidence interval [CI], 1.6-3.0, $\mathrm{p}<0.0001$ ), and HDL (OR 2.3, CI, 1.7-3.1, $\mathrm{p}<0.0001$ ). Strict control of triglycerides $<70 \mathrm{mg} / \mathrm{dL}$ resulted in less SVG occlusion. Conclusion: Uncontrolled triglycerides and high-density lipoprotein levels are the driving variables behind saphenous vein graft occlusion in patients who have undergone surgical myocardial revascularization. Maintaining triglycerides level $<70 \mathrm{mg} / \mathrm{dL}$ helps to prevent occlusion.

\section{Activation Sequence During Unifocal and Bifocal Atrial Pacing: New Observations and Insights in an Acute Canine Model}

Choudhuri I, MD, Aurora Cardiovascular Services

Krum D, MS, Aurora Electrophysiology Research

Agarwal A, MD, Department of Cardiology

Hare J, BS, Aurora Electrophysiology Research

Pinninti M, MD, Aurora Cardiovascular Services

Ahmad, AR, MD, Aurora Cardiovascular Services

Belohlavek, M, MD, PhD, Division of Cardiovascular Diseases, Mayo Clinic of Arizona

Background/significance: Alternate single- and dual-site atrial pacing for atrial resynchronization has been suggested to impact atrial fibrillation and diastolic heart failure that have been attributed 
in part to right atrial appendage (RAA) pacing, but clinical studies have not shown superiority of one approach.

Purpose: We studied atrial electrical activation sequence during unifocal and bifocal pacing to assess the atrial activation sequence during various pacing approaches.

Methods: Eight mongrel dogs $(29.4 \pm 1.4 \mathrm{~kg})$ were studied. Surface ECG leads I, II, V1, proximal, middle and distal coronary sinus recordings from a multielectrode catheter, and intracardiac recordings from bipolar plunge wires placed over the surface of the surgically accessed left atrium (LA) were monitored. Bipolar pacing was performed at 30-50 ms less than intrinsic sinus cycle length. Unifocal pacing was performed from the sinus node (SN), RAA, intra-atrial septal aspect of Bachmann's bundle (BBR), coronary sinus ostium (CSO) and perimitral low lateral left atrium (LLL). Bifocal simultaneous pacing was performed from RAA+BBR, RAA+CSO and RAA+LLL. Measured and derived intervals included activation time at non-paced sites in each atrium, intra-atrial activation time, global activation time, activation dispersion, activation dispersion index.

Results: RSinus node pacing homogenously activated the right atrium (RA); the left atrium (LA) was activated by anterior and posterior wavefronts simultaneously propagating septally to laterally and meeting at the lateral LA. RAA pacing reversed intra-RA activation and was associated with conduction delay and intra-atrial activation dispersion. Both RAA pacing as well as unifocal septal pacing from either the coronary sinus ostium or Bachmann's bundle (BBR) resulted in dispersion of septal activation, which then promoted dispersion of LA activation concordant with degree and pattern of septal activation. Lateral LA sites were activated by the first wavefront that arrived, whether anterior or posterior. Bifocal RAA+BBR pacing: 1) was associated with homogeneous RA activation, 2) preserved global atrial activation order, 3) did not produce LA activation dispersion, and 4 ) resembled the activation sequence of sinus node pacing.

Conclusion: Potentially deleterious effects of RAA pacing may arise from biatrial conduction delay and activation dispersion; these effects also may be seen with unifocal septal pacing; and such effects can be avoided by simultaneous bifocal pacing from RAA and BBR.

\section{Citations}

The following citations are for abstracts that were presented as part of the 2013 Aurora Scientific Day program. Since then, these abstracts were included in other publications.

Sud A, Catalano MF, Wham D, Guda NM. More evidence supporting genetic counseling and testing followed by endoscopic ultrasound in the early detection of pancreatic cancer in high risk patients. Gastroenterology 2012;142:S-616.

Nfor T, Shetabi K, Hassan W, Nfor Q, Khitha J, Gupta A, Bajwa T, Allaqaband S. Percutaneous coronary intervention versus coronary artery bypass grafting in high surgical risk patients with left main or three-vessel coronary artery disease. J Am Coll Cardiol 2013;61:E1706.
Mirza M, Strunets A, Holmuhamedov E, Sra J, Werner PH, Khandheria BK, Tajik AJ, Kress DC, Jahangir A. A novel histology based classification system to identify patients at risk of postoperative atrial fibrillation. Circ Res 2013;113:A038.

Lavine JA, Sang Y, Wang S, Ip MS, Sheibani N. Attenuation of choroidal neovascularization by $\beta(2)$-adrenoreceptor antagonism. JAMA Ophthalmol 2013;131:376-82.

Agarwal A, Nfor T, Cho C, Tyszkowska A, Ortiz D, Lazarov L, Palakuru S, Jahangir A, Allaqaband S. Gender-related differences in survival and predictors of survival for patients undergoing hypothermia after cardiac arrest. J Am Coll Cardiol 2013;61:E1570.

Mirza M, Yeneneh B, Strunets A, Cho C, Choudhuri I, Mortada ME, Nangia V, Bhatia A, Niazi I, Sra J, Jahangir A. Obesity is associated with lower mortality in patients with implantable cardioverter-defibrillator. Circulation 2013;128:A9400.

Kerl J, Spexarth F, Pedersen R, Stone M, Allaqaband S, Schulgit JL, Bajwa T, Gupta A, DeFranco AC. Beneficial effect of pointof-care bleeding risk calculator on anticoagulant selection and resource utilization: a pilot study Circulation 2013;128:A11295.

Emelyanova L, Mirza M, Jahangir A. Persistent atrial fibrillation is associated with selective reduction in rotenone-sensitive NADH oxidoreductase activity in human atria. Circulation 2012;126:A16125.

Agarwal A, Nfor T, Mirza M, Jahangir A, Gupta A. Safety and effectiveness of cardiac catheterization within 24 hours of admission in cardiac arrest patients treated with mild therapeutic hypothermia Circulation 2012;126:A11119. 